\title{
Cell Ultrastructure and Distribution of Trifluoroacetylated Protein-Adducts in Early Hepatic Injury in Mice Induced by Inhalation Anesthetic, Halothane.
}

\author{
Christine A. Brantner ${ }^{1}$, Mohammed Bourdi ${ }^{2}$, Mala Chakraborty ${ }^{2}$, Lance R. Pohl ${ }^{2}$, and Mathew P. \\ Daniels ${ }^{1}$. \\ ${ }^{1}$ Electron Microscopy Core Facility, NHLBI, NIH, Bethesda, MD. \\ ${ }^{2}$ Molecular and Cellular Toxicology Section, NHLBI, NIH, Bethesda, MD.
}

Halothane is an inhaled, general anesthetic that causes a rare form of severe liver injury by a two-step process. Initial exposure to halothane leads to formation of hepatic trifluoroacetylated protein-adducts (TFAPA) of this drug. These adducts cause hepatocyte injury by an unknown mechanism, resulting in their release into liver tissue and blood circulation, where they come in contact with cells of the immune system. In susceptible patients, TFAPA are recognized as foreign immunogenic proteins. After a second halothane exposure, the resulting liver injury is more severe due to an immune reaction against hepatic TFAPA. Characterization of ultrastructural changes in hepatocytes after initial exposure to halothane may help to elucidate the mechanism of injury as many drug-induced changes in ultrastructure have been observed in the liver [1].

The approach taken was to treat female Balb/cJ mice with a hepatotoxic dose of halothane. After 12 hours, a time point early in hepatotoxicity, liver pieces were removed from halothane treated and untreated mice. Pieces were chemically fixed for conventional transmission electron microscopy (TEM) to investigate any changes in subcellular ultrastructure resulting from the halothane dose. TEM images indicated ultrastructural changes in many hepatocytes. We observed differing amounts of structural damage in cells that are adjacent to one another. Hepatocytes from halothane treated mice contained more lipid droplets, a larger number of damaged mitochondria, dilated rough endoplasmic reticulum (RER) and a larger amount of smooth endoplasmic reticulum (SER) than hepatocytes from control mice. Various morphological changes indicated mitochondrial damage. These included small lucent regions in the matrix, overt swelling, dense flocculent bodies, septate mitochondria [2], separation of the inner and outer membrane and condensation of the mitochondrion (Fig. 1).

Pieces of liver were fixed, cryoprotected and frozen for cryo-immunogold labeling to localize TFAPA in the hepatocytes, using TFAPA specific antiserum [3]. Hepatocytes that appeared morphologically normal after the halothane treatment had sparse immunogold label over the entire cell indicating a low concentration of TFAPA (Fig. 2). In contrast, many hepatocytes showing ultrastructural changes were heavily labeled with gold over the endoplasmic reticulum and cytosol, indicating a high concentration of TFAPA in these organelles. These hepatocytes showed an increased number of lipid droplets and damaged mitochondria compared to lightly gold-labeled cells. Unexpectedly, the labeling was sparse over most of the mitochondria in hepatocytes with high concentrations of TFAPA.

Our results indicate that 12 hours after treatment with halothane, TFAPA are concentrated in several compartments in the damaged hepatocytes, but are at much lower concentrations in mitochondria. This suggests the possibility that the mechanism of early hepatocyte injury caused by halothane may be initiated by TFAPA formation in other organelles. 


\section{References:}

[1] MJ Phillips, S Poucell, J Patterson, and P Valencia, (1987) The Liver: An Atlas and Text of Ultrastructural Pathology (Raven Press, NY).

[2] H Kinagawa, Y Amakata, and T Maeda, Journal of Applied Toxicology vol 13(5) (1993), p. 327.

[3] PJ Hayden, T Ichimura, DJ McCann, LR Pohl, and JL Stevens, Journal of Biological Chemistry vol 266 no 28 (1991), p. 18415.

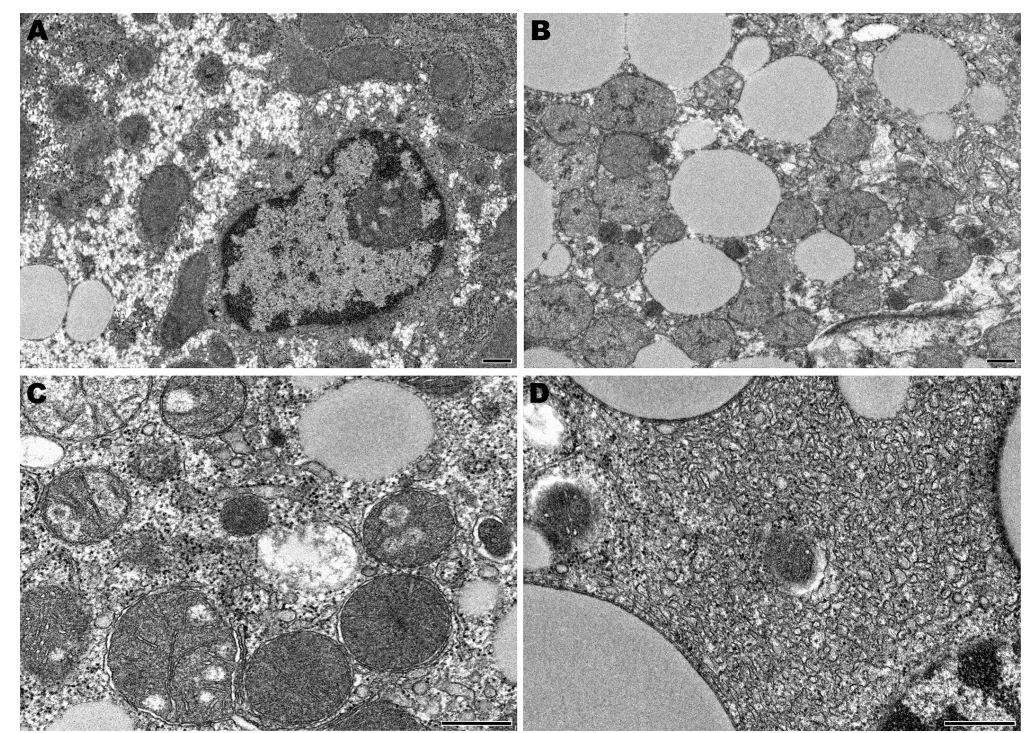

Figure 1. Resin infiltrated liver biopsy tissue. A. Untreated mice. B-D. Halothane treated mice. These hepatocytes show mitochondria containing dense flocculent material (B), increased lipid droplets (B), dilated RER (C), more SER (D), lucent regions in mitochondrial matrix (B\&C), mitochondrial swelling $(\mathrm{B} \& \mathrm{C})$, septate mitochondria (B), separation of the inner and outer membranes of the mitochondria (C) and condensed mitochondria (B, C, D). Bar $=500 \mathrm{~nm}$.
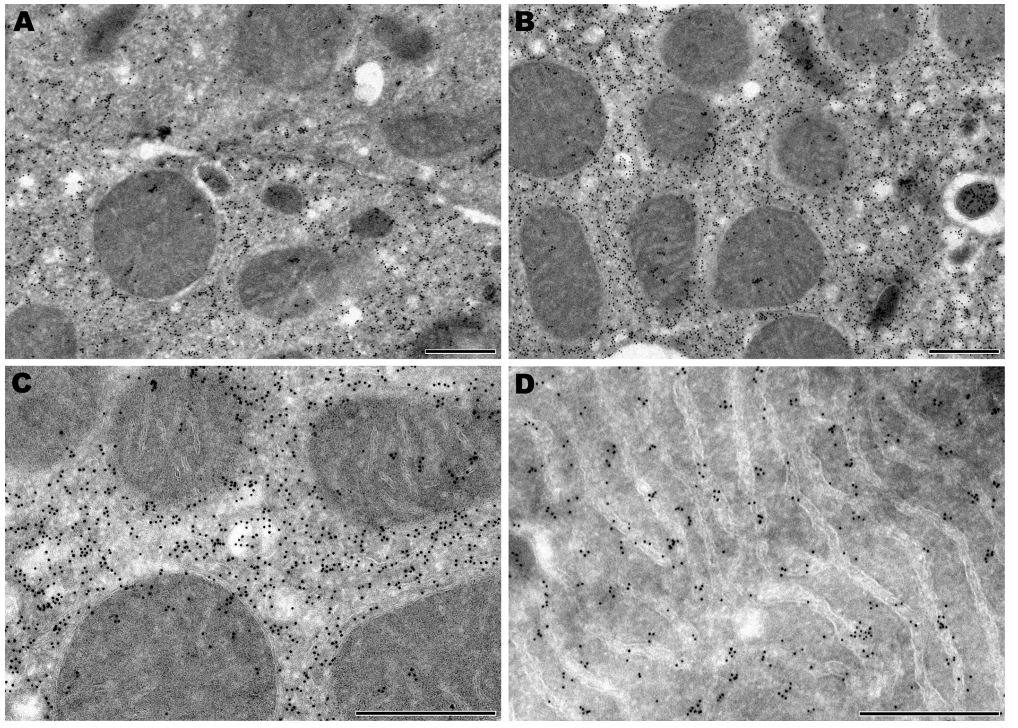

Figure 2. Cryo-immunogold labeling of halothane treated mice. A. Two adjacent cells with differing levels of TFAPA label. B \& C. TFAPA gold label is high over the endoplasmic reticulum and cytosol, but not over mitochondria. D. TFAPA label over RER. Bar $=500 \mathrm{~nm}$. 